\title{
¿Una rima perdida de Gustavo Adolfo Bécquer?*
}

\section{A Lost Rima by Gustavo Adolfo Bécquer?}

\author{
Luis Caparrós Esperante \\ Universidade da Coruña \\ luis.caparros@udc.es \\ ORCID iD: https://orcid.org/0000-0002-7583-6721
}

\section{RESUMEN}

El artículo da cuenta del hallazgo de un poema atribuido a Gustavo Adolfo Bécquer, publicado en el número 5 del periódico soriano Juventud, con fecha de 10 de enero de 1910. Para justificar esta atribución, se estudian primero las circunstancias que enmarcan la publicación, coincidente con la estancia allí de Antonio Machado, quien dejó testimonio de algún autógrafo becqueriano hasta hoy perdido, como lo haría posteriormente Gerardo Diego. En segundo término, se estudia el texto en busca de coincidencias que lo liguen al conjunto de la obra lírica de Bécquer.

Palabras Clave: Bécquer; Rimas; rima desconocida; poesía fúnebre; poesía decimonónica.

\begin{abstract}
The article reports the finding of a poem attributed to Gustavo Adolfo Bécquer, published in number 5 of Juventud, newspaper published in Soria, January 10, 1910. In order to justify this attribution, the circumstances surrounding the publication are studied in first place. The date coincides with Antonio Machado staying there. He left testimony of some Bécquer's autographs until today lost, as Gerardo Diego would do later. Secondly, the text is analysed in search of coincidences that could link it to the set of Bécquer's lyrical work.
\end{abstract}

Key words: Bécquer; Rimas; Unknown rima; Funeral poetry; Poetry of the 19th Century.

${ }^{*}$ Agradezco a D. José Ignacio Brieva Delgado el aviso y primera información sobre este poema. El trabajo presente se beneficia de la ayuda concedida por la Xunta de Galicia a Grupos con Potencial de Crecimento (referencia GPC2015/028), dentro del Grupo de Investigación Hispania. 


\author{
i.....! \\ Vivió quien aquí reposa \\ cual los que me escuchan viven. \\ ¡Cuán útil lección reciben \\ solo al mirar esta losa! \\ La vida más prodigiosa \\ La mejor felicidad \\ Solo hay de verdadero \\ Sepulcro y Eternidad.
}

Gustavo Adolfo BÉCQuer

Se trata de una octavilla con una extraña rima (abba:ac่dc்), aunque similar a otras con versos sueltos, como «Amor eterno» (abcb:adad), no recogida tampoco en las Rimas ${ }^{1}$. En este caso, y a diferencia de otros textos atribuidos a Bécquer en época reciente, el poema aparece con su nombre al final, aunque desnudo de cualquier explicación sobre su origen, contexto o circunstancias ${ }^{2}$. Comenzaremos, entonces, por aclarar estas últimas.

El poema apareció impreso, con fecha de 10 de enero de 1910, en el número 5 de la publicación soriana Juventud, que al llegar a ese número se presentaba ya como «Periódico decenal de Literatura, Ciencias y Artes». Entre sus «redactores honorarios» aparecían en primer lugar el político liberal D. José Canalejas y Méndez, D. Miguel de Unamuno, rector de la Universidad de Salamanca, D. Vicente Gay, catedrático de la Universidad de Valladolid, y D. Arturo Pérez Camarero, secretario del Ateneo de Valladolid. Seguían otros nombres de menos realce, aunque fuesen figuras destacadas de la vida local: un ingeniero agrónomo, un comandante, dos farmacéuticos y el director de $L a$ $\operatorname{Verdad}^{3}$. Este último era el político republicano y radical Benito Artigas Arpón, director de ese periódico (1909-1911) cuando Machado residía en Soria (Núñez Jiménez 2014: 78). Según los datos que encuentro, Artigas aparece ligado a diferentes publicaciones de signo progresista, hasta ser director de $\mathrm{La} V o z$ de Soria entre 1922 y 1936 (Checa Godoy 1989: 131).

${ }^{1}$ El poema, tal como aparece en la publicación, ofrece dudas en cuanto a su transcripción, especialmente en la segunda cuarteta. Para este trabajo he preferido respetar la (falta de) puntuación, sea o no debida a la voluntad del autor.

2 Pienso en las primeras ediciones de Los Borbones en pelota, que ya en su última edición excluye de la cubierta cualquier referencia a los Bécquer (Burdiel 2012), o en periódicas atribuciones de textos con pseudónimo (como Porras 2010), alguno de los cuales ha acabado siendo traducción del mismísimo Byron (Molina 2007).

${ }^{3}$ Figuran ahí Doroteo Relaño, ingeniero agrónomo, Domingo Ramos Ordóñez, comandante de infantería, Santiago Aparicio, doctor en Farmacia, Samuel Berdones, farmacéutico, y Benito Artigas Arpón, director de La Verdad (Juventud 1910: 1). 
El director de Juventud, la publicación que ahora nos interesa, era Miguel Ángel Ugena, un personaje joven cuya caricatura aparece en primera plana sosteniendo bajo el brazo una especie de palma acabada en plumilla goteante de tinta, sobre libros, facturas y botellas varias. En el número 2, de noviembre del año anterior, se daba cuenta de su nombramiento como presidente honorario del Comité Juvenil Republicano de Gómara (Juventud 15/11/1909: 3). Ugena había colaborado en La Verdad y en el número 3 de la nueva publicación, en diciembre de 1909, acababa un artículo con la triple invocación: «Viva España con honra! ¡Honra con libertad! Libertad de conciencias!». En el mismo número, la redacción declaraba venir «a sustituir a otra juventud que no tenía la virilidad necesaria para cumplir su cometido» (Juventud 1/12/1909: 1). Los nombres de la redacción, toda gente joven, como proclaman a cada paso, aparecen relacionados con diversas actividades culturales de la ciudad. Con todo, la vida de Juventud fue corta, de 1909 a 1910, al menos en Soria (Enciso Recio 2007). Desde 1912, y hasta 1913, la publicación pasaría a editarse en Murcia, como órgano de la sociedad Camino de Arte. Tendría entonces el mismo carácter decenal y el mismo director, al menos en un primer momento (González Castaño 1996: 24), lo que sugiere que Ugena hubiese marchado de la ciudad castellana con la cabecera bajo el brazo.

Si bien los datos anteriores no dan ninguna pista que conduzca a Bécquer, no resulta extraño que en una población pequeña como la Soria de entonces los poseedores de memorabilia becqueriana, vinculados de un modo $\mathrm{u}$ otro a Casta Esteban, su mujer, la hiciesen llegar a los periódicos o a los personajes del mundo intelectual que recalaban allí por una u otra razón. Este es el caso de Antonio Machado y lo será, años más tarde, de Gerardo Diego. Un dato relevante para nuestro caso es que la vida del periódico coincide con la estancia de Antonio Machado. Machado había llegado a Soria en 1907 y sigue allí cuando se publica este poema, en enero de 1910. En diciembre de ese año pasará a París, gracias a la beca de la Junta de Ampliación de Estudios, y allí será donde Leonor enferme. En agosto de 1912, muerta Leonor, el poeta abandonará por fin la ciudad.

Destaco este hecho porque es precisamente Antonio Machado quien nos da una pista probable para la atribución del poema a Bécquer. En los cuadernos de Los complementarios, bajo la fecha de 1923, recuerda la siguiente anécdota:

Conocí en Soria (1908) a un señor Noya, que fue el segundo marido de la madre de la mujer de Bécquer. Este señor Noya me regaló, como presente de bodas, dos autógrafos de Bécquer, dos composiciones inéditas que seguramente Bécquer no hubiera publicado. Yo las quemé en memoria y en honor del divino Gustavo Adolfo. Este señor Noya, suegrastro de Bécquer, era viejísimo y debe haber muerto ya (Machado 1998: 1260).

Le debía de fallar la memoria a Machado, pues el nombre real era don Manuel Zoya, viudo de la muy longeva Antonia Navarro Gonzalo, suegra de 
Bécquer, que había muerto con 95 años precisamente en 1908, dos años antes de la publicación del poema ${ }^{4}$. Pero esos datos poco importan para nuestro fin. Más curioso es que bastantes años después continuaba el trajín con los autógrafos becquerianos. Gerardo Diego da cuenta de una experiencia semejante en 1943, esta vez a propósito de otros dos autógrafos, uno de los cuales, «La gota de rocío», ya había sido publicado por José Gestoso y Pérez en $1886^{5}$ :

\begin{abstract}
Alguna o algunas (rimas) mejor hubiera sido dejarlas inéditas como sin duda lo habría hecho el poeta a tener tiempo de disponer él mismo su testamento lírico a la posteridad. A mis manos ha llegado también, y por el mismo conducto que esta Gota de rocio, otra composición en verso conservada en dos variantes autógrafas y que yo no me creo en derecho a imprimir. Si cualquier persona, aunque sea poeta, y admirable poeta, tiene derecho a desahogar en verso y para uso familiar o individual su intimidad herida, esto no quiere decir que tales documentos privados, por el simple hecho de su forma métrica, puedan lícitamente ser publicados si no contribuyen a esclarecer siquiera matices de su talento de artista poético o de artífice métrico, o al menos a iluminar puntos obscuros de su biografía (Diego 1943) ${ }^{6}$.
\end{abstract}

Aquí surge la pregunta esencial. ¿Y si ambos autógrafos, los de Machado y los de Diego, fuesen los mismos? Porque me inclino a pensar que aquella quema fuese más simbólica que otra cosa.

En alguna ocasión he sentido la tentación de atribuir al propio Gustavo alguno de los poemillas de poetas apócrifos que siguen a la cita anterior de don Antonio, en Los complementarios. No habría mejor salvoconducto para respetarlos sin herir, no obstante, la memoria del divino Gustavo Adolfo. Aceptando la lectura humorística que propone Machado (1998: 1272), hay uno que nos tienta sobre cualquier otro: «Tiburcio Rodrigálvarez. Nació en Almazán en 1838. Murió en Soria en 1908. Fue amigo de Gustavo Adolfo Bécquer, de quien conservó siempre grato y vivo recuerdo». No reproduciré aquí el fragmento de romance que transcribe, desde luego poco becqueriano y poco o nada ridículo, a diferencia del que Machado habría condenado al fuego. Esta poesía, nos informa después Machado, «fue leída por su autor, que poseía también algunos autógrafos de Bécquer» (ibíd.: 1273).

${ }^{4}$ Encuentro el dato, entre otros lugares, en el periódico Tierra Soriana, de 21 de enero de 1908, p. 3: «En la madrugada del día 19 del corriente falleció, en esta ciudad, la señora $\mathrm{D}^{\mathrm{a}}$ Antonia Navarro Gonzalo. La finada, a pesar de sus 95 años de edad, disfrutaba relativamente de excelente salud. [...] A su esposo D. Manuel Zoya, administrador del señor conde de Lérida, y a su numerosa familia acompañamos en el sentimiento que les embarga por pérdida tan dolorosa».

${ }_{5}^{5}$ Véase el comentario de la rima en la edición del Centro Virtual Cervantes (Caparrós 2002): http://cvc.cervantes.es/obref/rimas/rimas/rima_x_06.htm

${ }^{6}$ Para referencias más amplias y detalladas sobre este asunto, véase Caparrós Esperante (1997: 448). 
Ahí lo tenemos de nuevo. Gustavo Adolfo Bécquer habría ido dejando por tierras sorianas un largo rastro de autógrafos. El nuestro, por tanto, tiene muchas posibilidades de coincidir con cualquiera de los referidos por Machado o Diego y suma a ello la suficiente credibilidad, avalada por rúbrica, año y lugar, entre otras circunstancias vistas.

El lector que haya llegado hasta aquí probablemente concuerde con don Antonio en que el presente poema bien podría merecer el fuego. No lo pongo en duda, ni defenderé sus hipotéticas virtudes, pero en honor del divino Gustavo considero, o creo, que estos versos malamente pueden dañar su reputación. Con todo el respeto a los ilustres poetas citados, estoy convencido, como el amable lector, de que Bécquer está a cubierto de cualquier peligro por ese lado. A fin de cuentas, ¿no hay en las Rimas, como también en la obra de otros grandes poetas, versos o poemas enteros que malamente resisten la confrontación con los mejores? A cambio, nuestra curiosidad e interés literario agradece cualquier nuevo atisbo sobre el taller de un poeta como él.

El poema propone una enunciación en voz alta. Podemos imaginar la escena. La voz poética se dirige a «los que me escuchan» y lo hace, además, en un espacio bien definido: «al mirar esta losa». El pronombre, en su función deíctica, no deja dudas. El poema se está diciendo en un cementerio, ante la tumba de alguien querido. El fallecido o la fallecida, como el texto deja claro, no es ni mejor ni peor que quienes asisten a ese último tramo. El memento mori adquiere así algo de emblema o de vanitas barroca: «Eram quod es, eris quod sum» (yo era lo que tú eres; tú serás lo que soy). El muerto reposa «cual los que me escuchan viven». Vida y muerte se confunden o se equiparan. Esa es la «útil lección» que nos envía.

"Así como solo se halla la piedra del sepulcro entre la eternidad y la vida», escribe Chateaubriand (1806:179) en Genio del cristianismo. El sepulcro vendría a ser, según esta lectura canónica, una suerte de puerta que comunica la vida terrestre con la eternidad cristiana, esa puerta que debe atravesar el buen creyente para alcanzar la bienaventuranza. ¿Es esta también la perspectiva del poema que nos ocupa? No parece tan claro. Encontramos aquí la misma ambigüedad, ya de por sí significativa, que en otros poemas de Bécquer. Recordemos, sin ir ahora más lejos, la desolación de aquellos conocidos versos: «¡Dios mío, qué solos / se quedan los muertos!!» (rima 71, LXXIII) ${ }^{7}$. Los dos versos con que comienza la segunda cuarteta de nuestro poema son ciertamente positivos:

La vida más prodigiosa

La mejor felicidad

\footnotetext{
${ }^{7}$ Cito las Rimas en adelante por la edición del Centro Virtual Cervantes (Caparrós Esperante 2002): http://cvc.cervantes.es/obref/rimas/rimas/rima_x_06.htm
} 
Pero esa certeza se torna irónica al contacto con los dos últimos:

Solo hay de verdadero

Sepulcro y Eternidad.

En la práctica, el adverbio «solo» restringe de manera radical la extensión de tan posible felicidad. Incluso la anula, más allá del sepulcro. Lo verdadero no sería eso, sea vida prodigiosa o mejor felicidad, sino el Sepulcro mismo, o sea, la Muerte. Y junto a ella o por encima de ella, la Eternidad a secas. Establezcamos, con todo, una cautela, antes de proseguir nuestra lectura. Podría afirmarse que toda interpretación bascula sobre el significado que queramos dar a esa última palabra del poema: Eternidad. Sin duda, para un creyente será positiva, esto es, significaría la recompensa a los sufrimientos de esta vida terrenal, en lectura si se quiere manriqueña. En cambio, para quien no lo es, o al menos no claramente, como parece el caso de Bécquer, ese último verso es desolador: solamente permanecería la eternidad del sepulcro, sin atisbo de consuelo. Al menos, yo no observo ese consuelo o esa promesa de felicidad en ninguna parte del poema, leámoslo como lo leamos o asumamos cualquiera de esas perspectivas.

$\mathrm{Si}$ acudimos a las rimas conocidas, comprobaremos cómo tal lectura es viable. La 71 (LXXIII), cuyo estribillo citaba líneas arriba, expresa sin ambages la duda religiosa, lo que habría de ganarle todo tipo de reproches por los sectores eclesiásticos y conservadores de Sevilla, y no solo en ella, sino en el resto del país (Palenque 2009):

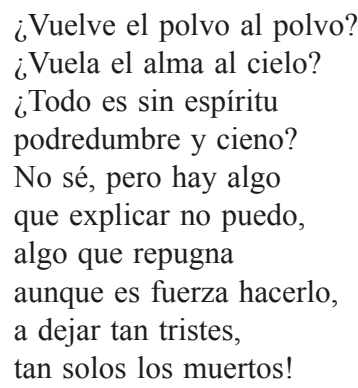

Si acudimos al final de la rima 74 (LXXVI), estaremos a salvo del fácil patetismo de la anterior, pero su conclusión es más desoladora aún, si cabe:

De aquella muda y pálida mujer me acuerdo y digo:

¡Oh, qué amor tan callado, el de la muerte!

¡Qué sueño el del sepulcro, tan tranquilo!

Y ya para concluir, ¿qué decir del final de la rima 25 (VIII)?: 
En el mar de la duda en que bogo

ni aun sé lo que creo.

En todo caso, no es momento para dilucidar las ideas de Bécquer al respecto, sino solo de mostrar la coherencia de estos versos con otros reconocidos como suyos. Mas queda aún una vuelta final, un detalle nada nimio que en una lectura rutinaria pudiera pasar desapercibido. Hablo del título del poema. Son cinco puntos indudablemente suspensivos, enmarcados por signos de exclamación. ¿Qué son esos cinco puntos sino la imagen visual, fáctica, de la nada? No es «iNada!», tan declamatorio. Es la propia nada, el vacío, como exclamación: «i.....!».

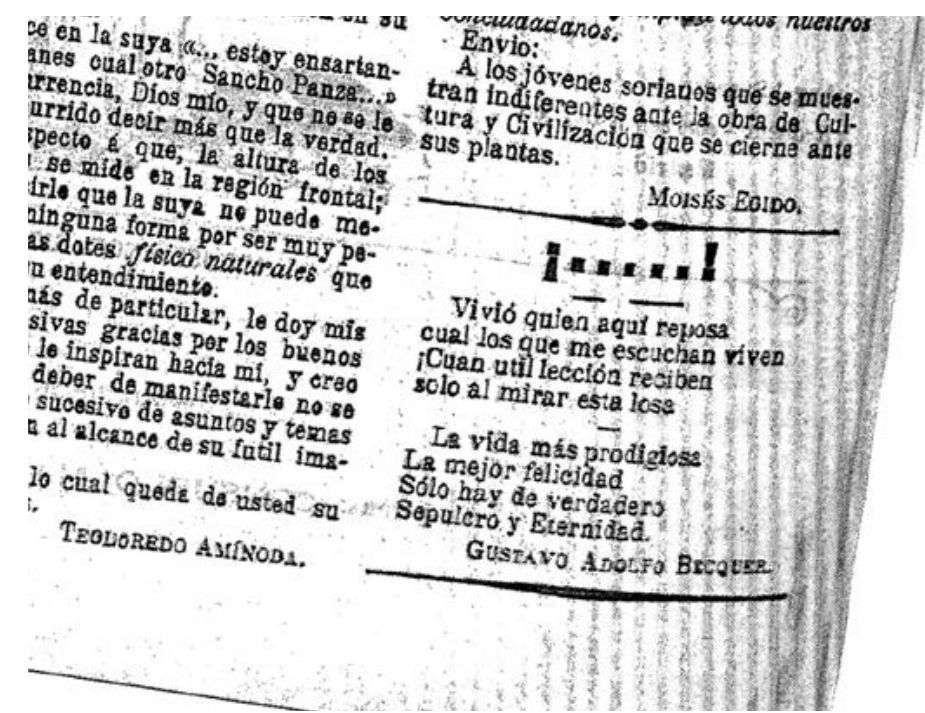

FiguRA 1. El poema en la publicación original, Juventud, 5, p. 2 (10 de enero de 1910).

\section{FUENTES}

Juventud. Revista quincenal de Literatura, Ciencias y Artes. 2 (15 de noviembre de 1909). Juventud. Revista quincenal de literatura, ciencias y artes. 3 (1 de diciembre de 1909). Juventud. Periódico decenal de Literatura, Ciencias y Artes. 5 (10 de enero de 1910). Tierra Soriana. Periódico independiente. II, 130 (21 de enero de 1908), p. 3.

\section{BiBLIOGRAFÍA CITADA}

Burdiel, Isabel (ed.) (2012). SEM, Los Borbones en pelotas. Zaragoza: Institución Fernando el Católico. 
Caparrós Esperante, Luis (1997). «Bécquer. Estética del borrador», Bulletin Hispanique. 99 (2), pp. 437-455.

Caparrós Esperante, Luis (ed.) (2002). Gustavo Adolfo Bécquer, Rimas de Gustavo Adolfo Bécquer. Alcalá de Henares: Instituto Cervantes, Centro Virtual Cervantes. Accesible en: $<\mathrm{http}: / / \mathrm{cvc}$.cervantes.es/obref/rimas/>

Chateaubriand, Francisco Augusto (1806). Genio del Cristianismo o Bellezas poéticas y morales de la religión cristiana. Madrid: Ibarra.

Checa Godoy, Antonio (1989). Prensa y partidos politicos durante la II República. Salamanca: Universidad.

Diego, Gerardo (1943). «Una rima inédita de Bécquer», La Nación. 7 de marzo. [Cita tomada de Robert Pageard, «Introduction» a Rimas de Gustavo Adolfo Bécquer. Madrid: CSIC (Clásicos Hispánicos), 1972, p. 28].

Enciso Recio, Luis Miguel (comis.) (2007). Exposición Antonio Machado en Castilla y León. Segovia-Soria, 2007. Valladolid: Junta de Castilla y León.

González Castaño, Juan (coord.) (1996). La prensa local en la región de Murcia (17061939). Murcia: Universidad de Murcia.

Machado, Antonio (1988). Poesía y Prosa. III. Prosas completas (1893-1936). Madrid: Espasa-Calpe.

Molina, Margot (2007). «El "bécquer" era de Lord Byron. Una obra del autor inglés se atribuyó en España al poeta sevillano», El País (Sevilla). 17 de enero.

Núñez Jiménez, Myriam (2014). La vida musical en la ciudad de Soria a través de la prensa: 1900-1910. Tesis doctoral presentada en la Universidad de Valladolid.

Palenque, Marta (2009). «Fama y fortuna de Gustavo Adolfo Bécquer: la heterodoxia de las Rimas y el episodio del retrato de la Biblioteca Colombina», Bulletin Hispanique. 111 (1), pp. 165-193.

Porras, Agustín (2010). Nuevas rimas de Gustavo Adolfo Bécquer. Zaragoza: Olifante.

Fecha de recepción: 23 de agosto de 2018.

Fecha de aceptación: 8 de noviembre de 2018. 